Rapport - Société canadienne d'histoire de l'Église catholique

\title{
Le père Joseph-Étienne Guinard, missionnaire centenaire (1864-1965)
}

\section{Raymond Douville}

Volume 32, 1965

URI : https://id.erudit.org/iderudit/1007331ar

DOI : https://doi.org/10.7202/1007331ar

Aller au sommaire du numéro

Éditeur(s)

Les Éditions Historia Ecclesiæ Catholicæ Canadensis Inc.

ISSN

0318-6148 (imprimé)

1927-7075 (numérique)

Découvrir la revue

Citer cet article

Douville, R. (1965). Le père Joseph-Étienne Guinard, missionnaire centenaire (1864-1965). Rapport - Société canadienne d'histoire de l'Église catholique, 32, 55-66. https://doi.org/10.7202/1007331ar

Tous droits réservés (C) Les Éditions Historia Ecclesiæ Catholicæ Canadensis Inc., 1966
Ce document est protégé par la loi sur le droit d'auteur. L'utilisation des services d'Érudit (y compris la reproduction) est assujettie à sa politique d'utilisation que vous pouvez consulter en ligne.

https://apropos.erudit.org/fr/usagers/politique-dutilisation/ 


\section{Le père Joseph-Étienne Guinard, missionnaire centenaire (1864-1965)}

Dans les quelques vingt minutes qui me sont accordées pour la présentation de ce travail, il me faut résumer la vie d'un homme qui a vécu cent ans, dont les deux-tiers au moins ont été d'une constante activité.

Ceux qui ont connu le père Joseph-Etienne Guinard, oblat de Marie immacultée, savent qu'en si peu de temps il est impossible de rendre justice à l'ampleur de son ouvre. Je me bornerai donc à évoquer quelques aspects de son apostolat social.

Je sais que, de façon générale, la sage politique de la Société d'Histoire de l'Eglise catholique est d'inscrire à son programme des sujets se rapportant plus particulièrement à l'histoire religieuse locale et régionale. Celui-ci répond-il à ces exigences? Sans hésitation, je réponds : oui.

Le père Guinard est surtout connu comme l'évangélisateur des Indiens du Haut Saint-Maurice, plus particulièrement de la tribu des Attikamègues ou Têtes-de-Boule, dont le premier missionnaire fut le célèbre jésuite Jacques Buteux, mis à mort dans cette région.

L'énergique historien du royaume du Saguenay, Mgr Victor Tremblay, réclame le privilège d'inscrire le nom du père Guinard sur la liste des missionnaires de son territoire. Il a raison. A la demande de ses supérieurs, le père Guinard est venu évangéliser les tribus indiennes du Saguenay, particulièrement celle de la Pointe Bleue, sur les rives du lac Saint-Jean. Et ce, au cours de longs et périlleux périples, qui rappellent ceux des missionnaires des premiers temps de la colonie.

Joseph-Etienne Guinard naquit à Maskinongé le 16 octobre 1864 . Après six années d'études classiques au séminaire de Trois-Rivières, il joint la communauté des pères Oblats en 1887 , et est ordonné prêtre à Ottawa, en décembre 1891, par Mgr Thomas Duhamel.

Son obédience le destine aux missions de la baie James. Il doit aller bâtir avec le père Fafard et le Frère Grégoire Lapointe la première résidence permanente d'Albany. Puis son supérieur lui demande un exploit presque surhumain. Il s'agit d'aller établir un poste d'évangélisation à 400 milles plus au nord, à Weenisk, sur le versant ouest de la baie. L'année suivante, il fonde le poste d'Arrawapiscat, à mi-chemin entre Weenisk et Albany. 
Au cours de ses six années d'apostolat dans cette région, le père Guinard a connu pour la première fois l'atroce supplice de la solitude. Il passa jusqu'à onze mois sans voir un seul confrère prêtre. Les Indiens et les commis protestants de la compagnie de la Baie d'Hudson étaient ses seuls compagnons.

Rappelé en territoire civilisé en 1898, pour surveiller l'impression d'un catéchisme en langue crise, le père Guinard se rend à la résidence de Maniwaki. Il y rencontre le père Jean-Pierre Gueguen, perclus de rhumatismes à la suite d'années d'apostolat chez les Têtes-de-Boule du Haut Saınt-ĪMaurıce. Guınard, quı connait déjà la langue de ces indigènes, s'offre à le remplacer. Il y demeurera jusqu'en 1942. Il aura été missionnaire durant un demi-siècle.

Si nous croyons utile, au cours de ces pages, d'appuyer sur le côté social de l'apostolat du père Guinard, c'est qu'il a vécu l'époque particulièrement agitée de l'infiltration massive des Blancs chez les tribus indiennes de sa région. Les missionnaires se plaignaient déjà de l'exploitation des Indiens par la vieille compagnie séculaire qu'est la $\mathrm{Hud}$ son's Bay. La subtile politique de cette dernière sera bientôt dépassée.

C'est avec la Hudson's Bay toutefois que le père Guinard aura ses premiers démêlés. Et déjà se manifestent chez lui des dons qui, avec les années, s'affermiront : énergie, persuation, diplomatie.

Comme le savent tous les missionnaires qui ont eu à traiter avec elle, la Hudson's Bay est', en forêt, un mal nécessaire. Il faut la traiter avec un certain ménagement. Dans les longues randonnées d'été ou d'hiver à travers bois, dans les tempêtes, dans le dénuement, les postes qu'elle a semés un peu partout sont l'unique source de ravitaillement et souvent de salut. Comme la grande majorité des commis de ces postes sont de religion protestante, les missionnaires leur inspirent à la fois de la méfiance et de la crainte. Pour s'accommoder avec eux, il faut sans cesse exploiter à bon escient ces deux sentiments.

Etant encore novice dans son métier, le père Guinard est chargé en 1907, en plus des missions ordinaires du Haut Saint-Maurice, de celle du poste de Waswanipi, beaucoup plus au nord et d'accès difficile. Avant la construction du chemin de fer de l'Abitibi, la seule façon de ravitailler cet endroit était de se rendre à Fort Rupert, sur la baie James. "Waswanipi fut toujours un crève-cœur pour le missionnaire ", avait dit le vieux père Guéguen. Guinard, à son tour, va bientôt s'en convaincre.

Après avoir terminé les missions du St-Maurice, le père Guinard se rend à Waswanipi, pagayant et portageant par lacs et rivières, luttant contre la faim, la fatigue, les embûches et les maringouins. Quand en. fin il arrive à ce poste, personne n'est au rivage pour l'accueillir, comme c'était l'habitude. Intrigué, il se rend voir le gardien du magasin de la Hudson's Bay Company, M. Johnson. Ce dernier reste froid, il ne lui offre ni logement ni repas, comme cela se faisait toujours. 
- Ce jour-là, je n'ai pas dîné », notera plus tard le missionnaire, avec une certaine mélancolie, car il a pagayé sur le lac depuis le petit jour jusqu'à midi. Intrigué d'une telle attitude, il retourne, à l'heure du souper, voir le bourgeois de la compagnie pour lui demander des explications. Voici ce qu'il apprend.

Le missionnaire précédent, le père Lemoine, avait été très malhabile. Il rudoyait les Indiens qui allaient lui quémander de la nourriture. Il leur disait que s'ils étaient pauvres, c'était parce que la compagnie de la Baie d'Hudson les volait, qu'elle payait les marchandises à trop bas prix. En somme, le père Lemoine n'a cessé, durant tout son séjour à cet endroit, de soulever les Indiens contre la Compagnie.

Le père Guinard réussit à convaincre $\mathrm{M}$. Johnson de ses bonnes intentions. Mais il n'est pas au bout de ses peines. Les hommes ne sont pas encore revenus de leur voyage de chasse sur les bords de la baie James. En les attendant, il enseigne le catéchisme aux enfants, et les baptise. Un jour, une femme lui dit : « Nous venons à ta maison de prière, mais nos hommes, je crois bien, n'y viendront pas, car ils ont tous abandonné ta religion. »

C'était, hélas! la vérité. Bien plus, un Indien du groupe s'était mis en tête d'être pasteur. Il se faisait appeler * le roi de la prière ». Bien traité par les missionnaires protestants et les chefs de la Compagnie, il passait chez les siens pour être bien plus généreux que le pauvre missionnaire catholique.

Le père Guinard visita chaque année la mission de Waswanipi de 1907 à 1928. On ne peut manquer d'être touché de la sobriété avec laquelle il relate ses déboires au cours de ses années d'apostolat dans ce poste perdu.

- J'ai presque toujours gardé le même nombre de catholiques, une trentaine. Quand j'en perdais, des protestants se convertissaient. Je dois dire que je n'ai pas eu tous les avantages : trois pères de familles catholiques moururent, un ouragan renversa la chapelle (il n'y a pas de doute qu'on exploita la chose comme étant un châtiment de Dieu). Le fanatisme protestant et la H.B.C. étaient contre moi. Puis vint résider un ministre écossais du nom de Catlidge, il enseignait l'école et il bâtit une chapelle. Deux fois l'évêque protestant Anderson, de Cachram, vint visiter ses ouailles. Puis vint un chef de poste apostat, John Tsenhoff. Je lui envoyai son extrait de baptême pour lui donner des remords. Celui qui lui succéda, Stuart, me rendit malade en mettant quelque chose dans ma nourriture. A l'arrivée du ministre, la H.B.C., arborait le pavillon, mais elle l'arborait à mon départ. Chaque fois que je suis allé à Waswanipi, je fis mon petit possible. Je n'y allais pas pour l'argent, car les indiens ne me donnèrent absolument rien. Chaque année, $j$ 'attendais les indiens deux ou trois semaines. Une fois, je les attendis 52 jours, jusqu'à ce qu'il n'y eut plus de provisions. Ce n'est que quinze jours après mon départ qu'ils arrivèrent de Ruppert's House. \ 
En 1928, le père Guinard fut dans l'impossibilité de retourner à Waswanipi. Il demanda à son supérieur de placer cette mission sous l'autorité des missionnaires de l'Abitibi. Ces derniers ne s'y rendirent pas, sous prétexte qu'ils voyaient les indiens de ce poste à Senneterre quand ils venaient vendre leur fourrures à la compagnie.

- Je veux bien croire, lit-on dans les Mémoires, que le Père voyait les hommes, mais leurs femmes et leurs enfants n'étaient pas baptisés, ni catéchisés, ni confessés, ni communiés. » Et il termine, non sans mélancoliẹe - Ainsi s'effondra et s'abîma la mission de Waswanipi. "

En dépit des déboires qu'il y connut, on sent que la mission de Waswanipi est restée chère au coeur du missionnaire. La description qu'il donne de ce poste est une des plus touchantes de ses Mémoires.

C'est de ce poste aussi qu'il desservit la mission de la Pointe-Bleue, sur les rives du lac Saint-Jean. Car ces tribus, affirme-t-il, parlaient la même langue. Elles n'étaient tout de même pas tout à fait voisines. Le prédécesseur de Guinard note qu'il a pris huit jours pour aller d'un poste à l'autre.

Plus tard, le père Guinard confina son apostolat à la région proprement dite du Haut Saint-Maurice et de l'Abitibi, qui s'ouvrait pour de bon à la vie économique et industrielle : construction du chemin de fer Transcontinental en pleine forêt, entre La Tuque et la Sarre; construction du barrage Gouin, puis l'organisation systématique des chantiers par les grandes compagnies de papier.

Ses mémoires fournissent sur cette période des renseignements uniques et inestimables.

Sur l'arpentage du tracé du Transcontinental, il raconte des anecdotes savoureuses dignes d'un roman d'aventures. Dès le début, des Indiens furent engagés pour servir de guides et porter les bagages et les instruments. Comme aucun de ces fonctionnaires ne comprenait leur dialecte, les Indiens réclamèrent un interprète. Seul le père Guinard, qui était de retour à la résidence de Maniwaki, pouvait le faire. Il accepta. L'employé du gouvernement fédéral qui vint le chercher n'était pas bien aise de voir un prêtre se rendre compte de leur travail et surtout de leur train de vie. "Ayant été obligé de marcher dans les bois, raconte le père, il voulut m'égarer en me laissant et courant à toutes jambes. Il avait mal choisi son homme : quand, tout essoufflé, il se retourna, il fut étonné de me voir sur ses talons. Je n'avais qu'à tendre la main pour le pousser dans la rivière.»

Une autre fois, on commande à des Indiens d'aller ravitailler les employés qui se trouvaient au White Bear Lake. Ils parcourent des centaines de milles sans trouver cet endroit, puis reviennent au camp bredouille. * Nous avons monté tous les ruisseaux et traîné les canots jusqu'à leur source, raconta le chef du groupe, un métis. Nous avons 
monté dans les arbres pour voir au loin, crié et pas de réponse et pas de White Bear Lake. » Finalement on découvre qu'il s'agit du lac Manitobegans, qu'on peut atteindre en quelques heures. N'en connaissant pas le nom, le chef arpenteur l'avait baptisé White Bear Lake. Pendant ce temps, les employés de ce poste mouraient de faim.

Quand il arriva au poste numéro I, le missionnaire remarque qu'une syrienne s'y trouve déjà \& avec ses valises remplies de rubans, de mouchoirs, de cravates, de bagues, etc. ».

Il s'étonne aussi d'y voir des Indiens Tête-de-Boule qu'il avait connus à Weymontachingue. Il apprend qu'ils sont rendus là parce que la Hudson's Bay ne veut plus leur faire crédit, pour se venger de ce que les Bors résistent aux Anglais en Afrique du Sud! Petit trait de mœurs qui n'est pas sans intérêt.

Le missionnaire célébrait d'habitude la messe du dimanche dans des chapelles où il y avait des embryons de colonisation. On se le disait, on ne sait comment, à des milles à la ronde, et les gens s'y rendaient de partout.

Un dimanche, alors que le père revêtait ses habits sacerdotaux dans la petite sacristie, une chanteuse vint lui dire : * Mon père, ça va mal dans le chœur de chant. Nous sommes en grève. »

C'était vrai. La tribune du chœur était vide. Le prêtre se contenta d'une messe basse. Ce fut ainsi le dimanche suivant, alors qu'au prône le père Guinard fit la déclaration suivante : * Mes frères, si nous n'avons pas de grand-messe chantée, je serai obligé d'allonger l'instruction, de prêcher plus longtemps, car l'office du dimanche doit durer au moins une heure et demie." Et le père de conclure : "Je ne sais si les chantres ont eu peur d'être ennuyés de mon éloquence; toujours est-il que, dans la suite, nous n'avons plus eu de messe basse les dimanches de mission. "

\section{Les chantiers}

Le père Guinard applique lors des visites des chantiers la même psychologie à base de compréhension, de charité et de mansuétude.

Il écrit quelque part : «Il me coûtait toujours de partir pour les chantiers. \ C'est que l'atmosphère générale, à l'arriveee, n'était guère édifiante. Ces gens vivaient presque à l'état sauvage. Des concours de jurons, où certains faisaient preuve d'un riche vocabulaire; des histoires et des chansons bassements grivoises.

Peu à peu, le missionnaire imposa sa méthode. Ayant groupé autour de lui ceux qui lui semblaient les plus sages, - car tous ne goûtaient pas ces extravagances -, il leur parlait des inventions nouvelles : téléphone, lumière électrique, gramophone; des phénomènes célestes, des mouvements de la terre, des grandes nouvelles du monde, du changement des gouvernements, des lois nouvelles de la province. Il lisait aussi le récit de quelques miracles. Ces faits miraculeux ont fait des 
conversions parmi les blancs qui ne croyaient à rien. Peu à peu la curiosité groupait les gens près du père.

Le missionnaire avait souvent à trancher des discussions. Voici un exemple qu'il a noté. "Un vieux m'aborde et dit : * Mon Père, tous les gens ici m'appellent "mon oncle». Je ne suis pas leur oncle et je ne voudrais pas l'être. Si vous les connaissiez comme je les connais, ce sont des voyous, ça sacre, ça blasphème, regardez donc ces visages !...» Quand il eut fini de déblatérer contre ses neveux, je lui dis en tendant les bras : "Eh bien !, tous m'appellent a Mon père * et je suis content de les avoir pour enfants !" Ce fut, note le missionnaire - et on le devine - un éclat de rire général.

\section{Le barrage Gouin}

On n'a pas encore songé à faire l'historique de la construction du barrage Gouin. Il ne pourrait être complet sans l'utilisation des notes personnelles du père Guinard.

Le barrage devait inévitablement inonder des territoires où se trouvaient depuis toujours des réserves indiennes. Pour les gouvernements, soit fédéral, soit provincial, les Indiens comptaient pour peu. Au nom du progrès, il s'agissait simplement de les déplacer, de les transplanter ailleurs.

C'est alors que le père Guinard prit la situation en main. Il ne voulait pas s'opposer à la construction du barrage. Il en comprenait la nécessité. Par contre, il ne pouvait non plus laisser exploiter ses ouailles sans défense, et vivant sur un territoire qui leur appartenait depuis toujours.

Après de multiples démarches, de nombreux voyages à Québec, à Ottawa, à Montréal, il obtint en partie satisfaction. Des compagnies achetèrent le bois des réserves qui devaient être inondées. La chapelle d'Obedjiwan fut rebâtie aux frais de la Commission des Eaux courantes et aménagée grâce à des dons de compagnies forestières et de particuliers. Mais il fallut des mois de démarches avant' que le département des Affaires indiennes d'Ottawa et le gouvernement provincial s'enten. dent pour rebâtir les maisons des Indiens. Le missionnaire voyait avec effroi approcher les mois d'hiver, car les Indiens n'avaient pour s'abriter que de misérables tentes provisoires.

$\mathrm{Au}$ point de vue industriel, la construction du barrage Gouin allait modifier du tout au tout ce vaste territoire qu'était le Haut Saint-Maurice. Mais pour les habitants de la forêt, c'était une catastrophe. L'inondation causée par ce lac artificiel fit mourir des milliers d'animaux sauvages de toutes espèces; même le poisson, car l'eau fut longtemps contaminée par les détritus et les microbes du sol et des arbres ensevelis. Les rives du lac étaient inabordables, à cause des troncs d'arbres et des souches qui fermaient tous les passages. Pour aller d'une réserve à l'autre, il fallait faire un immense détour dans la forêt vierge. De plus, il fallut chercher de nouveaux territoires de chasse. 
La vie traditionnelle des Indiens était déjà totalement modifiée par les éléments de la nature, quand arriva en plus l'invasion massive de l'industrialisation de leurs territoires. Une compagnie de fabrication de papier s'installe à Sanmaur. Des chantiers de bûcherons poussent un peu partout. Des clubs de chasse et de pêche choisissent les endroits les plus proprices. Enfin, grâce à la facilité des communications, des Blancs viennent concurrencer les Indiens dans le seul commerce qui leur apporte des revenus : la traite des fourrures, particulièrement le castor.

Bref, les Indiens se voient déjà acculés à la famine, et bientôt menacés d'extermination.

\section{Une délégation d'Indiens à Québec}

Fatigué de ces exactions et des injustices dont ses ouailles sont continuellement victimes de la part des Blancs, le père Guinard prend une résolution énergique. Il écrit au premier ministre de la province, l'honorable Alexandre Taschereau, et lui demande une entrevue au nom des principaux chefs de la tribu. Selon ses propres termes, il veut " impressionner le premier ministre ». Ce dernier accepte.

Le récit du voyage de la délégation est un des plus colorés des mémoires du père Guinard.

Le groupe prend place à bord du train du Canadien National à la gare d'Oskelaneo, environ 150 milles au nord de La Tuque. Les compagnons du missionnaire sont : le chef Megwesh, Gabriel Awachish, Basil Awachish, Matio Satcia et le marchand John Midlidge. A la gare de Sanmaur, le chef Charles Pitekwi et le gros Louis Kokokoho se joignent à la délégation.

Un représentant de la compagnie de la Baie d'Hudson a réservé les chambres à l'hôtel Saint-Roch, à Québec. Le trajet de la gare du Palais à l'hôtel se fait à pied, et retient l'attention des passants. " Habitués à marcher dans les bois, note le père Guinard, parmi les obstacles, ils faisaient de longs pas, levaient haut les pieds, heurtant les piétons. L'un d'eux était chaussé de mocassins en juillet. »

L'entrée des Têtes-de-Boule à l'hôtel fit sensation. Ils étaient venus vêtus de leurs habits des bois; ils portaient les cheveux longs, avaient le teint bronzé. Dès leur arrivée, ils vont en file s'asseoir à une table de la salle à manger. La nourriture qu'on leur apporte disparaît en un clin d'œil. L'un d'eux, le gros Louis, va rafler sans cérémonie le pain sur les tables voisines, au grand amusement des gens. On indique à un autre la porte de la cuisine. Il s'y rend et revient les bras chargés de victuailles. Des chambres leur ont été réservées. La plupart s'étendent par terre, car ils y dorment plus confortablement que dans les lits.

Le lendemain l'honorable Taschereau, accompagné du ministre Perrault, reçoit la délégation. Il avait été entendu qu'un des Indiens devait faire connaître les griefs en utilisant son dialecte, aussitôt interprété par le missionnaire. On croyait ainsi donner à l'entrevue une 
couleur locale et impressionner les représentants du gouvernement. Mais le Premier ministre ne goûte guère la mise en scène. Il laisse sa. voir sèchement qu'il est très occupé et veut au plus tôt mettre fin à l'en. trevue.

Alors le père Guinard prend la parole. Il va droit au but. Il ex. pose d'abord que les Indiens n'ont pour vivre que la chasse et la pêche. Le Premier ministre l'interrompt soudain :

- Pourquoi, dit-il, ne travaillent-ils pas comme les Blancs ? Il y a de l'ouvrage partout!

- Ces gens, répond le missionnaire, vivent dans les forêts loin de toute civilisation et où il y a peu d'employeurs. Puis, les montrant du doigt, il regarde le Premier ministre :

- Prendriez-vous, monsieur, l'un de ceux-ci pour serviteur?

Le père reprend son réquisitoire. Jamais il n'a été si éloquent. L'ignorance du Premier ministre de la condition de vie de ces Indiens l'a piqué au vif. Il sait qu'on l'écoutera et' qu'on le laissera aller jusqu'au bout.

Il développe deux points principaux : d'abord la façon de chasser de l'Indien et celle qu'utilise l'homme blanc. L'Indien connaît les mœurs des castors. A chaque barrage, il a soin de laisser un couple pour le repeuplement. L'homme blanc, par contre, tue tous les castors qu'il trouve. Pour aller plus vite, il fait sauter les barrages à la dynamite. Les castors qui ne sont pas tués fuient sous la glace et, leur gîte détruit, ils meurent de froid. De plus, les Blancs ne respectent pas les périodes de chasse et de pêche. Même les employés du chemin de fer et ceux du gouvernement, comme les arpenteurs et leurs employés, chassent à temps et à contre-temps, quand ils ne vont pas jusqu'à voler les pièges et les raquettes des Indiens.

Puis le missionnaire précise que les barrages faits par le gouvernement nuisent aussi aux territoires de chasse des Indiens. Le ministre Perrault l'interrompt :

- Mais allez-vous, dit-il, nous empêcher de faire des barrages ?

Le père Guinard bondit : «Je lui lançai, écrira-t-il, des yeux qui voulaient dire : « Toi, tais-toi, car... En effet, j'avais à lui dire qu'avant de construire des barrages, le gouvernement ne faisait pas couper le bois, que cela était un gaspillage, que des millions d'arbres tombaient dans l'eau et des millions de dollars étaient ainsi perdus, et cela pour gonfler la bourse des compagnies étrangères et sans doute de quelques ministres.»

Le missionnaire parla sur ce ton pendant près d'une heure. Il énuméra les droits qu'ont les Indiens sur leurs terres de temps immémorial. Puis il termine par ces mots : "Honorable Premier, ces Indiens ne viennent pas vous demander de garder indéfiniment leurs territoires de chasse. Mais en attendant que la civilisation les touche, ils 
vous demandent de les protéger contre les chasseurs blancs qui gaspillent leur avoir et les réduisent à une honteuse mendicité, à la famine. Honorable Premier, ces Indiens, même avec leurs droits, sont sans défense et sans protection. Ils ne font de mal ni opposition à personne. Il appartient à votre gouvernement de les défendre, de prendre la part de ces faibles en faisant des lois capables de remédier à leurs longues plaintes, à leurs réels griefs, et de faire observer ces lois par des moyens qui sont en votre pouvoir.

Le représentant de la Hudson's Bay à Weymontachingue, John Midlige, demanda la parole pour dire que tous les Indiens étaient endettés envers sa compagnie et qu'ils seraient dans l'impossibilité de payer leurs dettes et de vivre une vie humaine, si les Blancs continuaient à chasser sur leurs terres. Le père Guinard notera dans ses Mémoires que M. Taschereau fut fort impressionné des chiffres fournis par le marchand Midlige sur le nombre de peaux de castor achetées des Indiens et sur la quantité achetée des chasseurs blancs.

Avant de partir, note le père Guinard, le Premier ministre se leva, et lui présenta la main en disant : a Il est difficile d'aller contre ce que vous avez dit. *

Malgré cette note d'encouragement, les délégués s'en retournèrent avec peu d'espoir.

Le père Guinard écrira que a les Indiens attendirent encore un an ou deux avant de jouir du bon effet de leur délégation. Je ne sais, continue-t-il, où les journalistes de Québec prirent informations. En tout cas les journaux que j'ai lus n'expriment pas le pourquoi de la délégation des Indiens. De plus, j'ai passé dans leurs articles pour le père Etienne Blanchin. .

\section{Les recensements}

Le recensement décennal obligatoire par tout le Canada inclut aussi les Indiens. Parce qu'ils connaissaient mieux les familles, les missionnaires ont toujours accepté de bon gré cette tâche. D'abord, elle leur apporte un supplément de revenu intéressant; et aussi le recensement leur donne l'occasion de voir et de visiter les protestants non indiens qui vivent sur le territoire. Il arrivait qu'en demandant non seulement à quelle religion mais à quelle secte ils appartenaient, plusieurs ne le savaient pas, ou ne voulaient pas le dire. Etaient-ils méthodistes, presbytériens, baptistes? Les missionnaires l'apprenaient parfois en demandant quelle église leur père ou leur mère fréquentait dans leur village. Un jour, le père Guinard reçut cette réponse : \& Je suis un damné protestant !,

Les missionnaires connaissaient aussi l'époque favorable à ce travail. Une fois, un fonctionnaire laïque d'Ottawa fut chargé de recenser les tribus indiennes de l'ouest de la baie James. Il revient à Albany dé- 
couragé. J'ai parcouru, dit-il, des centaines de milles, et je n'ai vu que des femmes et des enfants. Il ignorait que c'était le temps de la chasse et que les hommes étaient partis. Il lui fallut plus tard refaire tout le parcours.

Dans les recensements indiens, nous apprend encore le père Guinard, outre la population, l'âge, le sexe, le nombre de familles et de feux, le gouvernement exigeait aussi de dire combien de loutres, de visons, de castors, de renards, de pécans, en un mot combien d'animaux ̀े fourrure rhaque rhasseur avait tuéc C.e qui était difficile à obtenir. car les Indiens n'ont pas l'habitude de compter leurs peaux et encore moins d'en dire le nombre. Ils comptaient sur leurs doigts, et il fallait accepter à peu près le chiffre qu'ils donnaient, ou le faire vérifier par le commis du magasin de la compagnie de la baie d'Hudson.

Les renseignements de toutes sortes obtenus lors des recensements étaient très précieux pour les missionnaires. Ce travail leur permettait d'avoir une bonne vue d'ensemble de la vie des blancs en forêt, et de l'influence de leur comportement sur les mœurs des Indiens.

C'est après avoir pris sa retraite, en 1942, que le père Guinard entreprit la rédaction de ses mémoires. Il compulse les notes griffonnées par-ci par-là tout au long de ses cinquante années de missions, il fait appel à ses souvenirs, et écrit plus de trois cent pages, qui forment un document absolument unique sur les événements dont il a été témoin. Il s'est toujours refusé à ce que ces notes soient publiées et même utilisées de son vivant.

\section{Le style des MEMOIRES}

Les MEMOIRES du père Guinard sont écrits dans une langue vivante, imagée, pittoresque, souvent primesautière. On y trouve des phrases que de grands écrivains se glorifieraient de signer. Voici quelques exemples. Quand le missionnaire entreprend son premier voyage vers la baie James, le paysage est monotone. Il note : « Le long du trajet, je regardais par la fenêtre; je ne voyais que souches, troncs d'arbres, chicots, broussailles et partout des pierres grosses comme un homme peut embrasser. Pas une maison, pas une grange, quelques petits ruisseaux qui achevaient de couler les eaux du printemps. La locomotive semblait se hâter pour éviter l'ennui. »

Un peu plus loin, il raconte qu'en compagnie du frère coadjuteur il a défriché un terrain pour semer des pommes de terre. "Sans le savoir, écrit-il, c'était l'endroit où d'Iberville avait hiverné après avoir chassé les Anglais de la baie d'Hudson. De gros peupliers s'étaient emparés paisiblement de la place du fameux guerrier. »

Voici un autre exemple de description sobre et précise; "Les Indiens arrivaient en guenilles, quelques-uns couverts de mauvaises peaux de lièvre qui s'étaient noircies. Ils venaient du Nord. de 80 à 500 mil- 
les, et plusieurs, leurs canots étant trop petits pour les porter, avaient marché au bord de la mer. »

D'autres tableaux, comme la description de la réserve de Waswanipi, et surtout celle d'un vieux poste de traite abandonné, sont pleins de réalisme et de pittoresque et écrits dans une langue élégante et soignée.

Oubliant les difficultés de son apostolat, voici comme il voit le poste de Waswanipi lorsqu'il rédige ses mémoires : "Waswanipi est un vieux poste qui a conservé jusqu'à ce jour toute l'apparence des anciens postes de l'intérieur, maisons toutes petites, en pièces, avec toit à pic en madriers, deux petites fenêtres et vitres très petites et une porte en planche. Voilà pour la maison des serviteurs. La maison du chef de poste est plus grande, et elle a un peu de peinture aux portes et aux fenêtres. Le magasin leur ressemble : le temps s'est chargé de peindre tout cela en gris foncé et morne. Le printemps, le magasin est vide; il ne reste que quelques écheveaux de fil, des pièges et des hachettes rouillées. Quand les grands canots arrivent, le magasin s'emplit. Les serviteurs sont les premiers à choisir et à acheter les marchandises. Puis c'est le tour des Indiens qui murmurent de n'être pas traités comme les autres. $\gg$

Voici la phrase toute simple que lui inspire un vieux cimetière abandonné : * Chaque fois que nous avons passé devant ce cimetière, nous avons arrêté les avirons et enlevé le chapeau pour dire quelques Ave aux petites âmes qui ont laissé leur corps à Mikiskan.»

Les Mémoires du père Guinard sont à l'image de sa vie : ils sont sobres, simples, propres, francs. Je dirais même d'un esprit raffiné que de longues années en pays sauvage n'ont pu ternir.

Quand il prend sa retraite en 1942, il a 78 ans d'âge et 50 ans do vie sacerdotale et missionnaire. Dans sa paisible retraite de la résidence de sa communauté à Maniwaki, tout en rédigeant ses mémoires, il rend à ses confrères de menus services.

Il se refuse à mourir. Le voici rendu à 87 ans et à 60 ans de vie religieuse. Ce sera bientôt la fin, croit-on. Mais non. Il est encore alerte. Il met à point la rédaction d'un ouvrage qui sera publié sous le titre Les noms indiens de mon pays. Les années passent : 95 ans, 96,97 . Le père Guinard a atteint l'âge des records. Il est le plus vieil Oblat du monde. Le plus vieux prêtre encore vivant. Pour ces grands sportifs que sont les missionnaires, il n'y a pas de Temple de la Renommée, du moins en ce bas monde.

Les journaux s'emparent quand même de sa personne, car il est devenu " quelqu'un ». On compte ses jours, comme les secondes d'un champion de courses. Mais le vieux routier tient bon. Il abandonne enfin, le 31 janvier 1965, \& après une brève maladie », précise une note officielle. Il a vécu 100 ans, 3 mois et 15 jours. 
Quelques semaines avant sa mort, un journaliste de Trois-Rivières, Fernand Gagnon, a longuement causé avec lui. Il avait conservé toute sa mémoire, toute sa lucidité d'esprit. A l'issue de l'entrevue, le vieux missionnaire eut cette réflexion spontanée : « J'aurais voulu faire davantage; mais je n'ai pas eu le temps !»

Raymond DouvilLe, M.S.R.C., de la Société des * Dix *. 